\title{
Random matrix models for phase diagrams
}

\author{
B Vanderheyden ${ }^{1}$ and A D Jackson ${ }^{2}$ \\ ${ }^{1}$ Department of Electrical Engineering and Computer Science (B28) and \\ SUPRATECS, University of Liège, Belgium \\ 2 The Niels Bohr International Academy, The Niels Bohr Institute, Blegdamsvej 17, \\ DK-2100 Copenhagen Ø, Denmark \\ E-mail: B.Vanderheyden@ulg.ac.be
}

\begin{abstract}
.
We describe a random matrix approach that can provide generic and readily soluble mean-field descriptions of the phase diagram for a variety of systems ranging from QCD to high- $T_{c}$ materials. Instead of working from specific models, phase diagrams are constructed by averaging over the ensemble of theories that possesses the relevant symmetries of the problem. Although approximate in nature, this approach has a number of advantages. First, it can be useful in distinguishing generic features from model-dependent details. Second, it can help in understanding the 'minimal' number of symmetry constraints required to reproduce specific phase structures. Third, the robustness of predictions can be checked with respect to variations in the detailed description of the interactions. Finally, near critical points, random matrix models bear strong similarities to Ginsburg-Landau theories with the advantage of additional constraints inherited from the symmetries of the underlying interaction. These constraints can be helpful in ruling out certain topologies in the phase diagram. In this Key Issue, we illustrate the basic structure of random matrix models, discuss their strengths and weaknesses, and consider the kinds of system to which they can be applied.

PACS numbers: $12.38 \mathrm{Aw}, 74.25 . \mathrm{Dw}, 71.10 . \mathrm{Fd}$
\end{abstract}

\section{Introduction}

Determining the phase diagram of a system is a central issue in many areas of physics. Knowledge of the thermodynamic state of a system as a function of externally controlled variables (e.g., temperature, density, or magnetic field) often provides important macroscopic information and can serve to help in identifying correlations among its microscopic constituents. A further determination of the nature of these correlations, which can be a consequence either of local interactions or of the cooperative behavior of a large number of constituents, is central to understanding the physical laws that govern the system.

In this Key Issue, we discuss the thermodynamics of two seemingly different types of systems: strongly interacting matter at extreme temperature and density, and high- $T_{c}$ 
compounds (including cuprates and ferropnictides) at finite temperature and doping. Strongly interacting matter is governed by Quantum Chromodynamics (QCD). For QCD with two flavors of massless quarks, a chiral and/or deconfining phase transition is expected at a temperature of $T \sim 160 \mathrm{MeV}$ for zero quark chemical potential [1] or at lower temperatures for a chemical potential as large as $\mu \sim 300 \mathrm{MeV}$ [2]. In this latter regime, attractive quark interactions may lead to a 'color-superconducting' phase [3, 4, 15, 6, 7, 8]. The high density end of the phase diagram is relevant for the physics of dense stars [8, 9, 10]; the lower densities are relevant for relativistic heavy-ion collisions [11] where it is hoped that unambiguous signatures of this phase transition can be observed.

The energy scales appropriate for the description of high- $T_{c}$ materials are evidently much (i.e., roughly $10^{10}$ times) smaller. For low doping, these materials exhibit an antiferromagnetic phase (AF) with transition temperatures of $\sim 150 \mathrm{~K}$ to $400 \mathrm{~K}$. For higher doping, these materials exist in a superconducting phase (SC) with a transition temperature of tens of degrees kelvin. This schematic picture can be refined to obtain a very rich phase structure with coexisting magnetic and pairing correlations and a variety of complex phenomena including stripes and pseudogaps [12, 13, 14, 15, 16, 17]. The basic mechanism for pairing is not well understood, and identifying the manner in which AF and SC phases dominate or coexist in the competition for the same electrons may be helpful in eliminating certain scenarios [18, 19, 20, 21].

Both QCD and high- $T_{c}$ materials are characterized by strong correlations among their microscopic constituents, and there is no indication in either case of a suitable "small parameter" that could lead to a meaningful perturbative description. Both systems would appear to be too complicated to permit analytic descriptions. Even numerical approaches are either extremely challenging or impossible as a consequence of the fundamental fermion sign problem (e.g., See [12, 22, 23, 24]). For each of these systems, numerical investigations yield highly oscillatory integrals that can prevent their simulation using techniques based on importance sampling. Given this situation, it may be useful to begin from the observation that these phase transitions are associated with the breaking of a symmetry (e.g., the staggered magnetization of AF breaks the spin rotational symmetry of the system).

In this Key Issue, we describe how an approach based on random matrix theory (RMT) can provide useful information regarding the possible phase diagrams for a variety of systems. Central to this approach is the identification of relevant symmetries and their consequences for the macroscopic properties of the system.

The basic ideas of random matrix theory (RMT) were introduced by Wigner when studying the complex spectra of intermediate and heavy nuclei [25, 26, 27]. His rationale can be summarized as follows: Although the detailed properties of the lowest energy states of nuclei could be understood by means of model Hamiltonians, there was no hope that this approach could explain the excited resonances observed with neutrons of energy up to several hundred $\mathrm{eV}$. These states were far too numerous and too closely spaced to permit detailed modelling. It was deemed to be both possible and more meaningful 
to determine the statistical properties of an ensemble of states, such as their density in a given energy interval or the distribution of their energy spacings and widths. This was achieved by constructing Hamiltonians with a structure that was directly dictated by the quantum physics of nuclei, but which otherwise contained matrix elements that were drawn at random on an appropriate distribution. The statistical properties of the highly excited resonances were then obtained by performing suitable ensemble averages analytically.

The central elements of this construction were the random and the deterministic parts of the Hamiltonians. Here, the random part of the Hamiltonian was not intended to describe randomly fluctuating processes or statistical disorder. Randomness was rather used as a statistical instrument: One considered a large ensemble of nuclei, each having a different Hamiltonian, and determined the properties of a group of nuclei by computing ensemble averages. The deterministic part of the Hamiltonian provided constraints on the resulting statistical properties. Initially, this deterministic structure was that dictated by the time-reversal symmetry of the strong interaction, which implies that the Hamiltonian can be written as a real symmetric matrix. By choosing statistical distributions so that ensemble averages were representative of the vast majority of the nuclei considered, the resulting properties were shown to be independent of the detailed dynamics of the nuclear interactions. Only the underlying symmetries were important.

These early ideas have evolved considerably since, and random matrix theory is now applied in many branches of nuclear, condensed matter, and particle physics. Still, in each case, symmetry plays a central role. This Key Issue will focus on the application of random matrix theory to the study of phase diagrams in systems where different symmetries compete thermodynamically. As in the early work of Wigner, random matrix theory is well suited for this task when the considered systems exhibit many intermixing energy states whose detailed properties are largely unknown or very difficult to determine. Starting from model Hamiltonians constrained by symmetries and generic mechanisms, the results are expected to be broadly representative of an ensemble of systems respecting these constraints. Ensemble averaging will eliminate details specific to individual Hamiltonians, and the features of the resulting phase diagram should thus be sufficiently robust that they will emerge in "almost all" specific cases.

Before considering this specific application, we wish to clarify the context in which it has been developed. Nowadays, random matrix theory encompasses a vast domain of research which has been reviewed in many works. (A non-exhaustive list is available in [28, 29, 30].) The consequences of symmetry constraints can be studied at two different levels - microscopic and macroscopic. Microscopic investigations deal with the correlations of the eigenenergies of the system on the scale of the average spacing. Examples of RMT predictions at this level include the spectral properties of nuclear resonances, atomic energy levels, sound waves in quartz crystals, transmission modes in quantum dots, and the eigenvalues of the QCD Dirac operator. One of the most important microscopic results is universality. When RMT applies, eigenvalue correlations are not only dictated by the symmetries of the system but are also 
independent of the details of the statistical distributions used to generate the ensemble averages. (See, e.g., section 7 of [29]).

The second, macroscopic level of description deals with the consequences of the underlying symmetries on the global state of the random matrix system. This is the level of the models considered below. Here, the central quantity is the partition function, from which the phase competition can be determined as a function of the variables of the theory such as temperature or chemical potential. The resulting phase diagram is a direct consequence of the symmetries of the system, and it is of interest to study their robustness with respect to variations of the coupling constants of the theory. At this stage, it is useful to note one detail of the random matrix approach: Matrix elements are drawn independently in such a way that all basis states are treated equally. Such 'democratic treatment' naturally implies that random matrix models are mean-field models.

These two levels of description are closely related to one another. For instance, in QCD with two massless flavors, it is observed that the smallest eigenvalues of the Dirac operator in vacuum accumulate near the origin of the spectrum. This accumulation is directly related to the spontaneous breaking of the chiral symmetry, as expressed by the Banks-Casher relationship which provides a direct connection between the average spectral density near the origin and the chiral order parameter [31]. Another strong relationship can be seen in the fact that many macroscopic properties are 'inherited' from symmetry constraints imposed at the microscopic level. As will be shown below, the random matrix partition function near the critical lines can be expanded as a power series in the order parameters. In this sense, RMT bears a strong resemblance to Ginsburg-Landau theory. In contrast to the Ginsburg-Landau approach, however, the coefficients in the random matrix expansion cannot be freely chosen but must satisfy relationships resulting from the form of the microscopic random matrix interactions. These constraints can preclude the occurrence of some symmetry breaking patterns. Thus, RMT can be used to determine the minimum number of symmetries which must be imposed at the microscopic level in order to reproduce a given phase structure.

In this Key Issue, we describe the basic steps required to construct random matrix models for phase diagrams. In the interests of readability, we have not attempted to provide technical details. Emphasis is rather placed on the ongoing simplification that arises in this construction, from the many variance parameters and complicated block structure needed to describe the random matrix interactions at the microscopic level to the few parameter ratios and elementary functional forms that characterize the thermodynamic potential.

We start with two basic models in section 2. The first describes chiral symmetry breaking in QCD, which is related to a quark-antiquark order parameter of the form $\left\langle\psi^{\dagger} \psi\right\rangle$ (in Euclidean conventions). The second is the case of an attractive phonon-like interaction, which produces pairing states with an order parameter of the form $\langle\psi \psi\rangle$. In section 3, we summarize our previous work on QCD and high$T_{c}$ superconductivity [32, 33, 34, 35, 36, 37]. Although different in nature, these 
systems exhibit phase structures with striking similarities in their symmetry breaking patterns. In particular, we discuss the conditions required for the occurrence of either a tetracritical or a bicritical point, corresponding respectively to the presence or the absence of a phase with mixed broken symmetries. In section 4, this discussion is extended to a tentative model for iron arsenide compounds with an $s_{ \pm}$order parameter, and we discuss the possibility that this system can have a state with coexisting magnetic and superconducting correlations. Section 5 presents final remarks regarding the random matrix approach to phase diagrams as well as a selected list of open problems.

\section{Two basic models}

\subsection{Chiral symmetry breaking in $Q C D$}

As a first illustration of the random matrix approach, we consider a model for chiral symmetry breaking in QCD with two light flavors, leading to an order parameter of the form $\left\langle\psi^{\dagger} \psi\right\rangle$. Working in Euclidean space and assuming a zero vacuum angle, the QCD partition function for fixed quark chemical potential, $\mu$, and temperature, $T$, is given as

$$
Z(\mu, T)=e^{-\Omega(\mu, T) / T}=\int \mathcal{D} \psi^{\dagger} \mathcal{D} \psi \mathcal{D} A e^{-S_{E}},
$$

where $S_{E}$ is the Euclidean action

$$
S_{E}=\int_{0}^{1 / T} d x_{0} \int d^{3} x\left(\frac{1}{2 g^{2}} \operatorname{Tr} F_{\mu \nu}^{2}-\sum_{f=1}^{N_{f}} \psi_{f}^{\dagger}\left(\mathcal{D}_{\mathrm{QCD}}+m_{f}+\mu \gamma_{0}\right) \psi_{f}\right) .
$$

Here, $\psi_{f}$ and $\psi_{f}^{\dagger}$ describe quark and antiquark fields of mass $m_{f}$ and $N_{f}=2$ is the number of flavors. Quarks interact via the exchange of gluon fields,

$$
A_{\mu}=\sum_{a=1}^{3} T_{a} A_{\mu a}
$$

where $T_{a}$ are the generators of the $S U\left(N_{c}\right)$ color algebra (with $N_{c}=3$ throughout this section), and the coupling arises via the Euclidean Dirac operator

$$
\mathcal{D}_{\mathrm{QCD}}=\gamma_{\mu}\left(\partial_{\mu}+i A_{\mu}\right) \text {, }
$$

where the $\gamma_{\mu}$ are hermitean. ( $\gamma_{0}$ is the matrix coupled to the time derivative $\partial_{0}$.) The dynamics of the gluon fields is contained in the quadratic term, $\operatorname{Tr} F_{\mu \nu}^{2} /\left(2 g^{2}\right)$, where

$$
F_{\mu \nu}=\partial_{\mu} A_{\nu}-\partial_{\nu} A_{\mu}+i\left[A_{\mu}, A_{\nu}\right]
$$

the trace is carried over colors, and $g$ is a coupling constant. As described in the introduction, the resulting interactions are too complex to be solved exactly, and one must resort to approximations.

The random matrix approach starts from symmetry considerations: In the chiral limit, $m_{f} \rightarrow 0$, the QCD partition function is invariant under $\operatorname{SU}\left(N_{f}\right) \times \operatorname{SU}\left(N_{f}\right)$. Nevertheless, chiral symmetry is spontaneously broken to $\mathrm{SU}_{V}\left(N_{f}\right)$ in the vacuum [38]. In the early 90's, it was discovered that chiral symmetry breaking could be seen in the dynamics of the small eigenvalues of the Dirac operator under fluctuations of the gauge 
field configurations. It was already known from the work of Banks and Casher [31] that the chiral order parameter, $\left\langle\psi^{\dagger} \psi\right\rangle$, can be expressed as an accumulation of the eigenvalues near zero virtuality. (The actual relationship will be made explicit below in equation (2.8).) In 1992, Leutwyler and Smilga derived further sum rules for the spectrum of the Dirac operator, which are dominated by the eigenvalues near zero virtuality [39]. In 1993, Shuryak and Verbaarschot [40] introduced a random matrix model for chiral symmetry breaking, where the QCD partition function in vacuum is approximated as

$$
\begin{aligned}
Z_{\mathrm{RMT}}(\mu=0, T & =0)=\int \mathcal{D} W \prod_{f=1}^{N_{f}} \mathcal{D} \psi_{f}^{*} \mathcal{D} \psi_{f} \exp \left[\sum_{f=1}^{N_{f}} \psi_{f}^{*}\left(\mathcal{D}_{\mathrm{RMT}}+m_{f}\right) \psi_{f}\right] \\
& \times \exp \left(-n \Sigma^{2} \operatorname{Tr}\left[W W^{\dagger}\right]\right) .
\end{aligned}
$$

In this approach, it is expected that the spectrum of the Dirac operator, $\mathcal{D}_{\mathrm{RMT}}$, has the same properties as $\mathcal{D}_{\mathrm{QCD}}$ provided the model satisfies the same global symmetries as QCD. In particular, in the chiral limit $m_{f}=0$ the Dirac operator should anticommute with $\gamma_{5}$. Working in the basis of the eigenstates of $\gamma_{5}, \mathcal{D}$ must therefore have the block structure

$$
\mathcal{D}_{\mathrm{RMT}}=i\left(\begin{array}{cc}
0 & W \\
W^{\dagger} & 0
\end{array}\right),
$$

where $W$ is an $n \times n$ complex matrix which models the QCD interactions. The choice of complex elements corresponds to the chiral unitary ensemble (chGUE), which is relevant for fermions in the fundamental representation. There exists two other ensembles, the chiral orthogonal (chGOE) and chiral symplectic (chGSE) ensembles, which are discussed in 41.

The dynamics of the interactions are now materially simplified. The quadratic term $\sim F_{\mu \nu}^{2}$ in the action $(2.2)$ is replaced by drawing the matrix elements of $W$ on a Gaussian distribution with zero mean and inverse variance $\Sigma$. Hence, an ensemble average is made over gauge field configurations. The corresponding number of degrees of freedom is proportional to $n$, which serves as the volume of the system and is to be taken to infinity at the end of the calculations (i.e., the thermodynamic limit). Note that the matrix elements $W_{i j}$ are drawn on the same distribution for all $i$ and $j$. In other words, the random matrix interactions are independent of the choice of basis states. As a result, no spatial range can be specified. The only scale introduced is that of the interaction strength, $\sim 1 / \Sigma$.

Given the form of equation (2.7), the spectrum of $\mathcal{D}_{\mathrm{RMT}}$ has the desired properties. Because the Dirac operator anticommutes with $\gamma_{5}$, eigenvalues occur in pairs $\pm i \lambda$ with eigenstates $u_{i}$ and $\gamma_{5} u_{i}$, so that the spectrum is symmetric about $\lambda=0$. Chiral symmetry is however spontaneously broken in the ground state. The chiral order parameter can be obtained from the Banks-Casher relationship [31] as

$$
\left|\left\langle\psi^{\dagger} \psi\right\rangle\right|=\lim _{\lambda \rightarrow 0} \lim _{m_{f} \rightarrow 0} \lim _{n \rightarrow \infty} \frac{\pi \rho(\lambda)}{2 n},
$$


where

$$
\rho(\lambda)=\left\langle\sum_{i} \delta\left(\lambda-\lambda_{i}\right)\right\rangle
$$

is the average spectral density. Note that the different limits in (2.8) do not commute. They must be taken in the order indicated since eigenvalues accumulate near $\lambda=0$ as $n$ is taken to infinity. (In particular, it can be seen that reversing the $\lambda \rightarrow 0$ and $n \rightarrow \infty$ limits would give zero, since $\rho(0)=0$ for any finite $n$ as a consequence of the $\pm \lambda$ symmetry noted above.)

Further properties of the spectrum were shown to match those of QCD (for eigenenergies $\lambda \ll \Lambda_{\mathrm{QCD}}$ ). First, the model of equations (2.6) and (2.7) was shown to obey the QCD Leutwyler-Smilga sum rules [39, 42]. Second, direct comparisons with data from numerical simulations of QCD on a lattice showed that the spectrum of the Dirac operator simulated at the scale of the average energy spacing (the "microscopic level density') agrees with the random matrix predictions within computational uncertainties [43, 44, 45, 46, 47, 48,. All together, these results demonstrated that the eigenvalue correlations near $\lambda=0$ are a direct consequence of the underlying chiral symmetry and do not depend on the specifics of the interactions. In that sense, the correlations are universal. Of course, QCD could have been an "exceptional" theory with spectral correlators different from the ensemble average. The fact that it is not the case provides encouragement that random matrix arguments can also offer insight into its macroscopic properties.

Chiral symmetry breaking can be studied at a macroscopic level with the aid of the thermodynamic potential. The chiral random matrix model can be solved exactly by standard methods. There are three steps. First, the matrix elements $W_{i j}$ are integrated over to give [49]

$$
Z_{\mathrm{RMT}}(0,0)=\prod_{i=1}^{N_{f}} \mathcal{D} \psi_{i}^{\dagger} \mathcal{D} \psi_{i} e^{Y}
$$

where $Y$ is an effective quartic interaction potential given as

$$
Y=-\frac{1}{n \Sigma^{2}} \sum_{f, g, i, k} \psi_{L k}^{f \dagger} \psi_{R i}^{f} \psi_{R i}^{g \dagger} \psi_{L k}^{g}+\sum_{f, i} m_{f}\left(\psi_{L i}^{f \dagger} \psi_{L i}^{f}+\psi_{R i}^{f \dagger} \psi_{R i}^{f}\right),
$$

Second, each fermion bilinear is expressed as combinations of squares which can then be linearized by means of a Hubbard-Stratonovitch transformation

$$
e^{A Q^{2}} \sim \int d x \exp \left(-\frac{x^{2}}{4 A}-Q x\right),
$$

which introduces an auxiliary variable, $x$. Focusing on the term relevant for chiral condensation, only one such auxiliary variable, $\sigma$, is considered, and the partition function becomes

$$
Z_{\mathrm{RMT}}(0,0) \sim \int d \sigma e^{-2 n N_{f} \Omega(\sigma)}
$$

with a thermodynamic potential

$$
\Omega(\sigma)=\frac{\Sigma^{2} \sigma^{2}}{2}-\log (|\sigma+m|),
$$


where we have assumed $m_{1}=m_{2}=m$ for simplicity. Interestingly, the function $\Omega(\sigma)$ is even in the limit $m=0$, reflecting chiral symmetry. For the final step, the integral on the right side of equation (2.13) can be solved exactly in the thermodynamic limit $n \rightarrow \infty$ by the saddle-point method, as $\lim _{n \rightarrow \infty}\left(1 / 2 n N_{f}\right) \log Z_{\mathrm{RMT}}=-\min _{\sigma} \Omega(\sigma)$. In the chiral limit $m \rightarrow 0$, the minimum of $\Omega(\sigma)$ is simply given as

$$
\sigma_{0}=1 / \Sigma \text {. }
$$

The corresponding chiral condensate in vacuum is then

$$
\left\langle\psi^{\dagger} \psi\right\rangle=\lim _{m \rightarrow 0} \lim _{n \rightarrow \infty} \frac{1}{2 n N_{f}} \frac{\partial \log Z}{\partial m}=\Sigma .
$$

Note again that the thermodynamic limit must be taken first to produce a meaningful result. In conclusion, the vacuum state has a non-zero order parameter as a result of the spontaneous breaking of chiral symmetry.

This analysis can be extended to finite temperature and density in order to describe the complete phase diagram. Technically, $T$ and the chemical potential, $\mu$, are introduced in the Dirac operator by the fermion Matsubara frequencies $\omega_{n}=$ $\mu+(2 n+1) i \pi T$ (where $n$ is an integer). In other words, $\mathcal{D}$ is replaced by $\mathcal{D}+\gamma_{0} \omega_{n}$, and the partition function is summed over the $\omega_{n}$ 's [49, 2]. The thermodynamic potential can again be determined by following the procedure described above. The result is simply

$$
\Omega(\sigma)=A \sigma^{2}-\sum_{n, \pm} \log \left(( \pm(\sigma+m)-\mu)^{2}+(2 n+1)^{2} \pi^{2} T^{2}\right),
$$

where $A$ is a constant proportional to $\Sigma^{2}$. Note the structure of the logarithm term, which can be rewritten as $\sum_{n, \pm} \log \left(E_{ \pm}^{2}+(2 n+1)^{2} \pi^{2} T^{2}\right)$, where $E_{ \pm}= \pm(\sigma+m)-\mu$ represent the quark $(+)$ and antiquark $(-)$ single quasiparticle energies in a fixed auxiliary field, $\sigma$.

A simplified potential can be obtained by restricting the frequency sum to the two smallest frequencies, $\mu+i \pi T$ and $\mu-i \pi T$ [49]. The resulting potential can then be minimized analytically and gives a polynomial gap equation for $\sigma_{0}(\mu, T)$,

$$
A \sigma_{0}=\sum_{ \pm} \frac{\sigma_{0}+m \pm \mu}{\left(\sigma_{0}+m \pm \mu\right)^{2}+\pi^{2} T^{2}}
$$

which can be solved exactly in order to describe the phase diagram in the $(\mu, T)$ plane.

The resulting phase diagram is shown in figure 1 (a) in the chiral limit $m \rightarrow 0$. (The other phase structures will be discussed in section 3.) In vacuum, $\sigma_{0} \neq 0$ and chiral symmetry is spontaneously broken. For small values of $\mu$, chiral symmetry is restored at a finite temperature via a continuous (second-order) phase transition. By contrast, chiral symmetry restoration along the zero temperature axis is realized by means of a first-order phase transition, with discontinuities in both the pressure and the density of the system. In this respect QCD bears a strong analogy to an antiferromagnet, where an increase of the applied magnetic field induces an abrupt vanishing of the staggered magnetization of the ground state. In QCD, the chemical potential is an intensive quantity whose increase leads to a variation of the baryon density $\left\langle\psi^{\dagger} \gamma_{0} \psi\right\rangle$. However, 


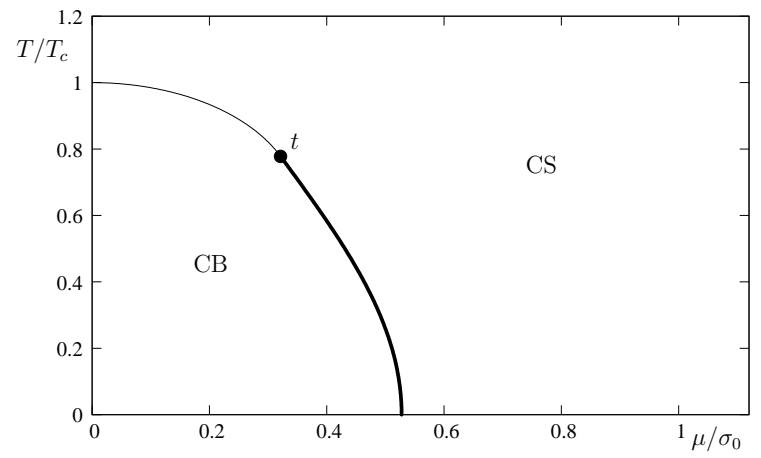

(a)

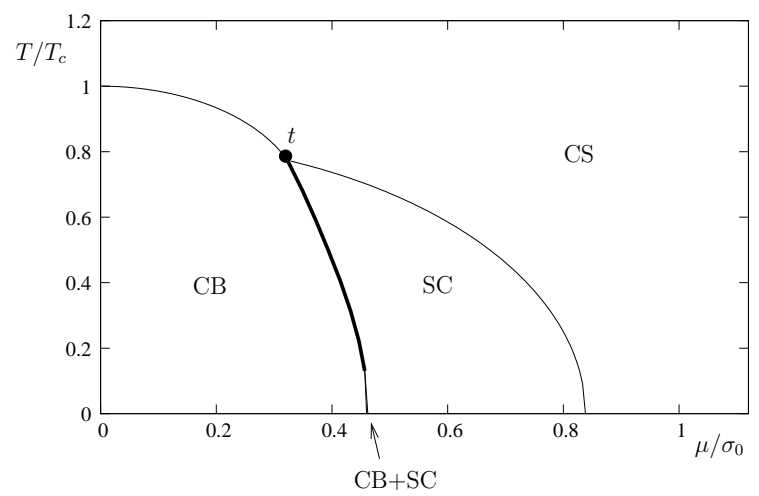

(c)

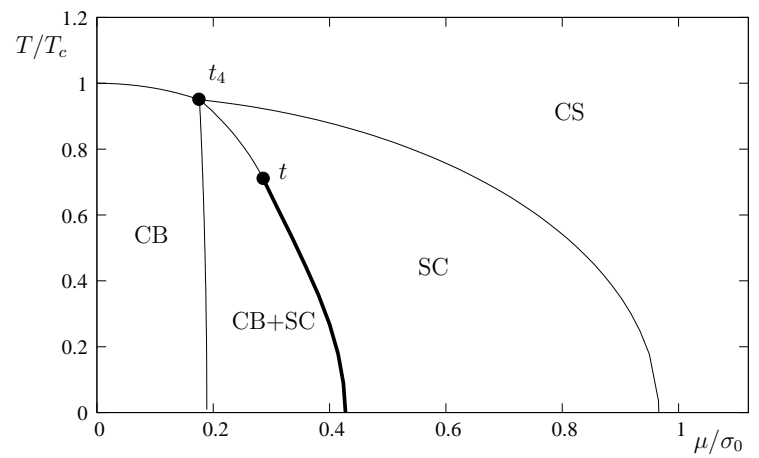

(e)

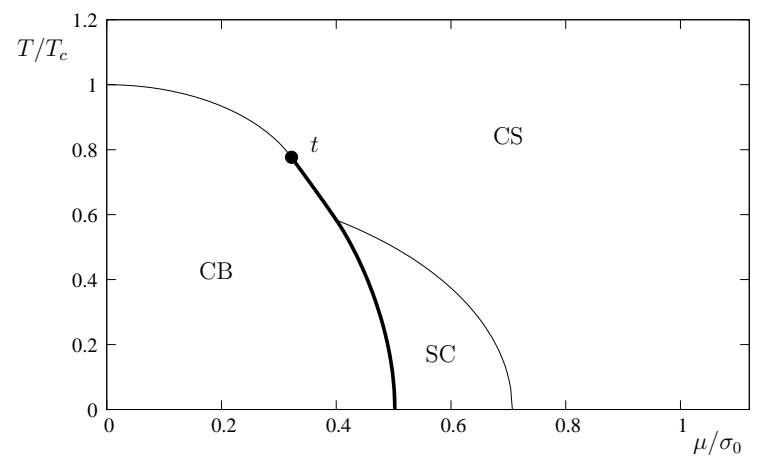

(b)

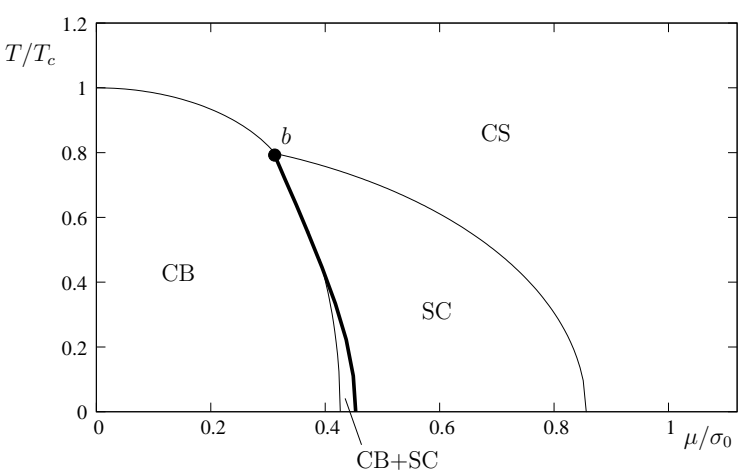

(d)

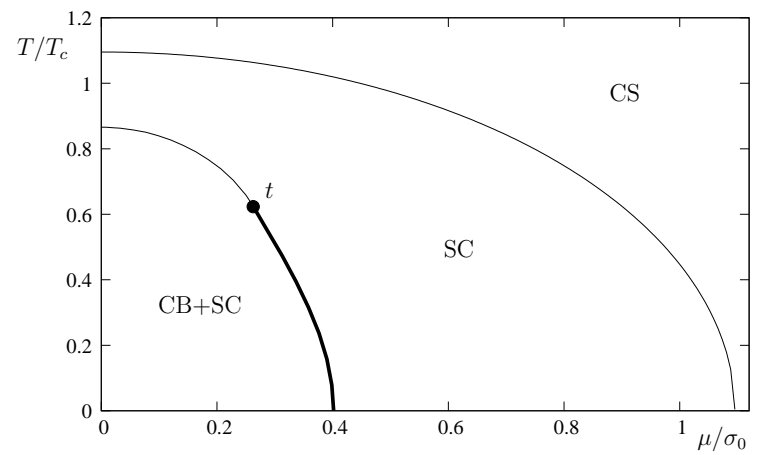

(f)

Figure 1. Figure (a) shows the phase diagram for the chiral symmetry breaking model of section 2. This phase structure also shows one of the six topologies that can be realized in the random matrix model for the competition between chiral symmetry breaking and quark pairing, see section 3 CB is the chiral broken phase, CS the chiral symmetric one, $\mathrm{SC}$ the superconducting phase, and $\mathrm{CB}+\mathrm{SC}$ the mixed broken symmetry phase. (This is a phase with an homogeneous coexistence of the two orders.) First- and second-order transitions are depicted by thick and thin lines, respectively. $t$ denotes a tricritical point, $t_{4}$ a tetracritical point, and $b$ a bicritical one. For the diagrams (a)-(e), $T_{c}$ is the transition temperature at $\mu=0$ and $\sigma_{0}$ is the value of the chiral field in vacuum. For diagram (f), $T_{c}$ and $\sigma_{0}$ assume the same expressions (i.e., $T_{c}=\sigma_{0}=\sqrt{3 / B}$ ) even though they no longer correspond to a transition temperature or a chiral condensate. From top left to bottom right, the panels correspond to $\alpha=0.3$, $\alpha=0.75, \alpha=1.054, \alpha=1.1, \alpha=1.4$, and $\alpha=1.8$. Note that the phase structure (c) contains a small wedge of mixed broken symmetry which is separated from CB by a second-order line and from SC by a first-order line. The figures are adapted from 33 . 
increasing $\mu$ also causes reduction of the chiral order parameter, $\left\langle\psi^{\dagger} \psi\right\rangle$. Similar to the abrupt vanishing of the staggered magnetization under an applied magnetic field, $\left\langle\psi^{\dagger} \psi\right\rangle \rightarrow 0$ through a first-order transition. The rest of the phase diagram must be continuously related to the transitions observed at $T=0$ and $\mu=0$. As shown in figure 1 (a), the second-order line starting from the $\mu=0$ axis continuously joins the first-order line starting from the $T=0$ axis. The two lines meet at a tricritical point, $t$, located at finite values of $T$ and $\mu$.

The macroscopic results are clearly of a different nature from those obtained at the microscopic level. It is important to understand the role played by the global symmetries at the macroscopic level. How good are the random matrix predictions for the phase diagram? Do they have a universal character? To answer these questions, two regions must be distinguished - near the critical lines and away from them.

In the critical regions, the random matrix potential of equation (2.17) resembles that typically obtained in a Ginsburg-Landau approach. Along the second-order critical line, $\Omega(\mu, T)$ can be expanded as a series of powers of $\sigma$ to give

$$
\Omega(\mu, T)=\Omega_{0}(\mu, T)+a(\mu, T) \sigma^{4}+b(\mu, T) \sigma^{6}+\ldots,
$$

where $a(\mu, T)$ and $b(\mu, T)$ are known functions of $\mu$ and $T$. In this form, random matrix theory resembles a mean-field $\phi^{4}$ theory. Furthermore, the coefficient $a(\mu, T)$ vanishes at the tricritical point, and the theory exhibits the critical exponents of a mean-field $\phi^{6}$ theory. This result would also have been obtained by a more traditional Ginsburg-Landau approach. It is important, however, to recognize that the construction of the random matrix model at the deeper microscopic level of the interactions fixes the coefficients $a(\mu, T)$ and $b(\mu, T)$ uniquely. This is not the case in Ginsburg-Landau theory. This fact has consequences on the thermodynamic competition between different phases and will be discussed further in section 3 .

Away from the critical regions, the shape of the phase diagram must be interpreted with care. The phase structure was established on the basis of a severely truncated sum over Matsubara frequencies. This is a rough approximation, which was shown to be equivalent to a mere rescaling of the temperature through a monotonic function [34]. (Note that this truncation is not necessary if the phase diagram is to be considered numerically.) Such a transformation shifts the phase boundaries and the critical points in the $(\mu, T)$ plane, but it preserves the overall shape of the phase diagram. Hence, the topology of the phase diagram - the relative positions of the different phases is a direct consequence of the underlying symmetry and is a robust prediction. The predicted order of a given phase transition is more delicate to assess. As stressed earlier, the random matrix approach ignores most of the dynamics of the interactions. A more detailed description can affect the energy balance between the different states of the system and lead to local modifications of the order of a phase transition, e.g. change a second-order line into a weak first-order one. This level of approximation is however perfectly acceptable as such corrections are to be expected in any mean-field approach. Indeed, the order parameter is weak in the critical regions, and it is thus fairly sensitive 
to the inclusion of additional thermal or quantum fluctuations.

\subsection{Phonon mediated superconductivity}

As a second example, we consider a random matrix model for a system of electrons with a longitudinal phonon interaction leading to a pairing condensate $\sim\langle\psi \psi\rangle$. This model will be compared to results expected from mean-field approaches based on the BCS theory in order to illustrate the method and to highlight differences with respect to the more complete microscopic approach. The random matrix model was motivated by a more extensive study including antiferromagnetism and superconductivity [37, the results of which will be discussed in section 3. Once again, some calculational details have been omitted in order to focus on the basic structure of the model.

We wish to reproduce the basic structure of the partition function

$$
Z_{\mathrm{ph}}(\mu, T)=\int \mathcal{D} \phi \mathcal{D} \psi^{\dagger} \mathcal{D} \psi e^{-\int_{0}^{1 / T} d x_{0}\left(H_{0-e l}-\mu N+H_{0-p h}+H_{\mathrm{int}}\right)},
$$

where $\phi(x)$ is a real field describing longitudinal phonons, $\psi$ describe electrons, $H_{0-e l}-\mu N$ is their free energy, $H_{0-p h}$ is the Hamiltonian of free phonons, and the electron-phonon coupling is given as (see, e.g., [50])

$$
H_{\text {int }}=g \sum_{\alpha} \int d^{3} x \psi_{\alpha}^{\dagger}(x) \psi_{\alpha}(x) \phi(x),
$$

with $\alpha$ the spin index and $g$ a real coupling constant. It is implicitly assumed that the phonon interaction decreases sufficiently rapidly with distance to justify the contact form of $H_{\text {int }}$. The phonon interaction is attractive and competes with Coulomb repulsion; for electrons near the Fermi surface, the phonon interaction dominates and tends to produce $s$-wave electrons pairs with an order parameter

$$
m_{S C-s}=\left\langle\sum_{\mathbf{p}, \omega_{n}, \alpha, \beta} \psi_{\alpha}\left(\mathbf{p}, \omega_{n}\right)\left(i \sigma_{2}\right)_{\alpha, \beta} \psi_{\beta}\left(-\mathbf{p},-\omega_{n}\right)\right\rangle,
$$

where $i \sigma_{2}$ is the antisymmetric spin matrix. Pairing leads to a reorganisation of the ground state of the system, and in particular, leads to the opening of a gap in the quasiparticle spectrum. The gap, $\Delta$, obeys the equation

$$
\Delta=\frac{|\lambda| T}{(2 \pi)^{3}} \sum_{n} \int d^{3} p \frac{\Delta}{((2 n+1) \pi T)^{2}+(\varepsilon(p)-\mu)^{2}+\Delta^{2}},
$$

where $|\lambda| \sim g^{2}, n$ are integers, and $\varepsilon(p)-\mu \approx v\left(p-p_{F}\right)$ is the energy of a single noninteracting electron near the Fermi surface. In this construction, it is implicitly assumed that the relevant states are centered around the Fermi energy, $\mu$, in a narrow shell with an energy width $\sim 2 \omega_{D}$ where $\omega_{D}$ is the Debye energy for longitudinal phonons.

A random matrix formulation would naturally start with a partition function of the form

$$
Z_{\mathrm{RM}-\mathrm{ph}}(\mu, T)=\int \mathcal{D} \psi^{\dagger} \mathcal{D} \psi \mathcal{D} H_{\mathrm{int}} P\left[H_{\mathrm{int}}\right] e^{-\psi^{\dagger} H \psi},
$$

where $H=H_{0}-\mu N+H_{\text {int }}$ is the sum of the single-electron Hamiltonian and an hermitean term $H_{\text {int }}$ describing the exchange of phonons. This term is chosen diagonal 
in spin space. The term $P\left[H_{\text {int }}\right]$ describes the dynamics of the phonon fields. In order to construct an order parameter of the form of equation (2.22), the following parametrization is introduced: the full set of states is divided in two complementary subsets $\{1, \alpha, i\}$ and $\{2, \alpha, i\}$ where the index $i$ denotes $\left(\mathbf{p}, \omega_{n}\right)$ in the first subset and $\left(-\mathbf{p},-\omega_{n}\right)$ in the second. With this parametrisation, the random matrix version of the order parameter assumes the form

$$
m_{S C-s}=\left\langle\sum_{i, \alpha, \beta} \psi_{1, i, \alpha}\left(i \sigma_{2}\right)_{\alpha, \beta} \psi_{2, i, \beta}\right\rangle .
$$

The interactions $H_{\mathrm{int}}$ are constrained by time-reversal symmetry. In coordinate representation, the phonon field is real since it carries no electric charge. In momentum representation, we thus require that the Fourier components of the matrix elements satisfy the complex conjugation constraint

$$
\left(\mathbf{p}, \omega_{n}\left|H_{\text {int }}\right| \mathbf{q}, \omega_{m}\right)=\left(-\mathbf{p},-\omega_{n}\left|H_{\text {int }}\right|-\mathbf{q},-\omega_{m}\right)^{*} .
$$

Using the parametrization just introduced, it can be shown that this is equivalent to imposing the following block structure [37] :

$$
H_{\text {int }}=\mathbf{1}_{\text {spin }}\left(\begin{array}{cc}
B & C \\
C^{*} & B^{*}
\end{array}\right),
$$

where the hermiticity of $H_{\text {int }}$ requires that $C$ is complex symmetric and $B$ is Hermitean. Because the form of the time-reversal operator depends on the basis of states used, the block structure of the random matrix $H_{\text {int }}$ is associated with a specific choice of basis of states (i.e., states in the momentum representation related by time-reversal symmetry).

As in our first example from QCD, we wish to explore the consequences of the block symmetry in equation (2.27) while considerably simplifying the dynamics of the phonon interactions. First, we assume that the important states are those for which phonon exchange dominates Coulomb repulsion, as in the microscopic theory. As a second approximation, we note that the detailed theory of equation (2.20) contains a free phonon term $H_{0-p h}$ which is a bilinear function of the field $\phi$. As in QCD, we replace this term by a random matrix with elements drawn on a Gaussian distribution with zero mean and fixed inverse variances, $\Sigma_{B}^{2}$ and $\Sigma_{C}^{2}$. This gives

$$
P\left[H_{\text {int }}\right]=\int \mathcal{D} B \mathcal{D} C \exp \left(-N\left(\Sigma_{B}^{2} \operatorname{Tr} B B^{\dagger}+\Sigma_{C}^{2} \operatorname{Tr} C C^{\dagger}\right)\right),
$$

where $N$ represents the total number of states (over the two subsets) and will be taken to infinity at the end of the calculations. This form introduces the possibility of having different strengths associated with scatterings from a region to itself $\left(\Sigma_{B}\right)$ and between different regions $\left(\Sigma_{C}\right)$. A particular choice can be made that reflects the known nature of the phonon interaction for any given material, but this is not essential for what follows. Except for this difference, no other scale is introduced. With the parametrization introduced above, the resulting interaction has an infinite range in momentum space and is thus a contact interaction in coordinate representation, as in equation (2.21). 
To proceed, we again restrict the sum over Matsubara frequencies to the two lowest terms, $\pm \pi T$. The free energy term becomes

$\psi^{\dagger}\left(H_{0}-\mu N\right) \psi=\sum_{ \pm, i, \alpha}\left(( \pm i \pi T-\mu) \psi_{1, \pm, i, \alpha}^{\dagger} \psi_{1, \pm, i, \alpha}+(\mp i \pi T-\mu) \psi_{2, \pm, i, \alpha}^{\dagger} \psi_{2, \pm, i, \alpha}\right)$,

where, consistent with the parametrisation introduced above, states in $\{1\}$ and $\{2\}$ have opposite Matsubara frequencies. (Here, the Matsubara index \pm is written out explicitly.) The random matrix model is solved following the three-step procedure described above: First, the interaction matrix elements are integrated over, yielding an effective potential $Y$ with quartic terms of the form $\sim \psi_{i}^{\dagger} \psi_{j} \psi_{j}^{\dagger} \psi_{i}$ and $\sim \psi_{i}^{\dagger} \psi_{j} \psi_{i}^{\dagger} \psi_{j}$. (Here, only the random matrix indices are shown.) The second set of terms, which describe an attractive interaction favoring the formation of pairing condensates $\left\langle\psi_{i} \psi_{i}\right\rangle$, are of particular interest. Second, the quartic terms are linearized by means of HubbardStratonovich transformations. Third, a thermodynamic potential is calculated exactly in the thermodynamic limit $N \rightarrow \infty$. The minimum of this potential describes the thermodynamic state of the system as a function of $T$ and $\mu$. The entire procedure is described in detail in [37]. Concentrating on the pairing channels, we find

$$
Z_{\mathrm{ph}-\mathrm{RM}} \sim \int d \Delta d \Delta^{*} \exp (-2 N \Omega)
$$

with

$$
\Omega(\Delta)=A|\Delta|^{2}-\frac{1}{2} \log \left(\mu^{2}+|\Delta|^{2}+\pi^{2} T^{2}\right),
$$

where the auxiliary field $\Delta$ is complex and is associated with the order parameter of equation (2.25) while the constant $A$ is given as

$$
A=\frac{1}{\Sigma_{B}^{-2}+\Sigma_{C}^{-2}} \text {. }
$$

Note the structure of $\Omega(\Delta)$, which is similar to that found for QCD in equation (2.17). It contains a quadratic term $A|\Delta|^{2}$ which represents the energy cost for creating a condensate and a logarithmic term which can be rewritten as $\sum_{ \pm} \log \left(E_{ \pm}^{2}+\pi^{2} T^{2}\right)$ where $E_{ \pm}= \pm\left(\mu^{2}+|\Delta|^{2}\right)^{1 / 2}$. This expression is reminiscent of the quasiparticle energies in the microscopic theory, $\pm\left(\left(\varepsilon_{p}-\mu\right)^{2}+|\Delta|^{2}\right)^{1 / 2}$ with $\varepsilon_{p}$ taken to zero. It is hardly surprising to see this limit emerge in the random matrix framework since no electron energy was introduced in the single-electron Hamiltonian $H_{0}$.

It is also interesting to consider the gap equation, which is given in the random matrix model as

$$
\frac{\partial \Omega}{\partial \Delta^{*}}=0 \quad \Longrightarrow \quad A \Delta=\frac{\Delta}{2\left(\mu^{2}+|\Delta|^{2}+\pi^{2} T^{2}\right)} .
$$

Comparison with the gap equation (2.23) of the microscopic theory shows evident similarities, but (2.33) is polynomial and can thus be solved exactly: A paired state with $\Delta \neq 0$ is found for $\mu^{2}+\pi^{2} T^{2} \leq 1 /(2 A)$, and the system is unpaired $(\Delta=0)$ otherwise. As a result, at a given density (i.e., at some fixed $\mu$ ), the system undergoes a continuous transition from a paired to an unpaired state at $T=T_{c}=\pi^{-1}\left(1 /(2 A)-\mu^{2}\right)^{1 / 2}$. For small gaps, $\Omega$ can be expanded as $\Omega(\Delta) \approx \Omega(0)+a(\mu, T)|\Delta|^{2}+b(\mu, T)|\Delta|^{4}$, where $a(\mu, T)$ and 
$b(\mu, T)$ are known functions. Near $T_{c}$, we have $a \sim\left(T-T_{c}\right)$, so that the theory gives the critical exponents of a mean-field $\phi^{4}$ theory.

The reader may be surprised that the random matrix gap equation (2.33) does not share a well-known feature with the BCS gap equation: In the zero temperature limit and for $\Delta \rightarrow 0$, the right side of (2.23) contains a logarithmic singularity for $\varepsilon(p)$ near the Fermi surface. As a result, a solution with $\Delta \neq 0$ exists no matter how weak the strength $\lambda$. In contrast, the random matrix gap equation admits a paired solution only if $\mu^{2}+\pi^{2} T^{2} \leq 1 /(2 A)$. It follows that weak interactions, corresponding to large inverse variances $\Sigma_{B}^{2}$ and $\Sigma_{C}^{2}$ as well as a large value of $A$, favor a paired state in only a restricted region of the $(\mu, T)$ plane and a vanishing gap in the rest of the phase diagram.

Comparing (2.23) and (2.33), the absence of a logarithmic singularity in the random matrix approach can be attributed to two factors: First, the truncation of the Matsubara sum, which prevents the construction of a Fermi-Dirac distribution, and second, the lack of an explicit reference to momentum in the right side of equation (2.33), which prevents the construction of a density of states over a range of energies. As a result, for fixed $\Delta$, the random matrix model contains only two energy states, $E_{ \pm}$, where a microscopic model would have energies $\varepsilon(p)$ continuously distributed near the Fermi surface $\mu$. The gap equation is then polynomial and does not contain the logarithmic singularity. The discrepancy with the BCS approach should not be considered as a weakness. The vanishing of the random matrix gap occurs in regions of parameter space where the condensate would otherwise be weak and fragile against the inclusion of additional fluctuations (either quantum or thermal). Determining the fate of the gap in these cases clearly requires going beyond mean-field, even in a BCS approach. The level of approximation offered by the random matrix approach thus appears reasonable.

\section{Phase diagrams in QCD and in high-Tc materials}

The nature of the correlations that arise when different symmetries compete for the same degrees of freedom and the macroscopic manifestations of this competition are matters of primary concern in condensed matter systems. The result is often a rich phase structure that can depend sensitively on the coupling parameters of the theory. We will now show how random matrix theory can be used to address these questions by considering two apparently different systems - QCD at finite temperature and density and high- $T_{c}$ superconductors and $d$-wave pairing as found in the cuprates.

For QCD, we consider the thermodynamics competition between chiral symmetry breaking, described in 2.1, and quark pairing, which is analogous to the electron pairing discussed in 2.2. Quark pairing might occur in dense and cold quark matter as a result of the attraction provided by the strong interaction in the color antitriplet channel [3, 4, 5, 6, 7]. The formation of quark pairs might lead to energy gaps as large as $\Delta \sim 100 \mathrm{MeV}$ for quark chemical potentials of the order of $300 \mathrm{MeV}$ and have direct consequences on the phase structure of nuclear matter at high density. In the following, we consider the case of QCD with two light flavors, for which the dominant 
pairing order parameter is given as

$$
\left\langle\psi_{f \alpha \sigma}(p) \psi_{g \beta \sigma^{\prime}}(-p)\right\rangle=\phi\left(p^{2}\right) \varepsilon_{f g} \varepsilon_{\alpha \beta 3} \varepsilon_{\sigma \sigma^{\prime}},
$$

where $p$ is a four-momentum, $f$ and $g$ are flavors, $\alpha$ and $\beta$ denote colors, while $\sigma$ and $\sigma^{\prime}$ are spins. The tensors $\varepsilon$ impose antisymmetric combinations of the various indices. This order parameter breaks color symmetry from $S U(3)$ to $S U(2)$ while preserving the $S U_{L}(2) \times S U_{R}(2)$ flavor symmetry.

For high- $T_{c}$ materials, we consider a two-dimensional system of electrons on a square lattice. There is a vast literature reporting a rich and complex phase structure arising from the competition between magnetic and pairing correlations [12, 13, 14. Proposed order parameters include $d$-wave pairing (see [51, 52] and references therein), stripes [15], or correlations arising from the pseudogap phenomenon [53, 54]. Here, we investigate the competition between two of these possibilities. The first one is antiferromagnetism with an order parameter of the form

$$
\mathbf{m}_{A F}=\left\langle\sum_{\mathbf{p} \omega_{n} \alpha \beta} \psi_{\alpha}^{\dagger}\left(\mathbf{p}+\mathbf{Q}, \omega_{n}\right) \boldsymbol{\sigma}_{\alpha \beta} \psi_{\beta}\left(\mathbf{p}, \omega_{n}\right)\right\rangle,
$$

where $\mathbf{p}$ are momenta in the first Brillouin zone, $\mathbf{Q}=(\pi \hbar / a, \pi \hbar / a)$ is the AF ordering vector ( $a$ is the lattice spacing), $\omega_{n}=(2 n+1) \pi T$ are fermion Matsubara frequencies, $\alpha$ and $\beta$ are spin indices, $\boldsymbol{\sigma}$ are the spin Pauli matrices, and $\langle\ldots\rangle=\operatorname{Tr}\left(\ldots e^{-\beta H}\right)$ denotes a thermal average. The second order parameter is associated with $d$-wave pairing and is given as

$$
m_{S C-d}=\left\langle\sum_{\mathbf{p}, \omega_{n}} g(\mathbf{p}) \psi_{\uparrow}\left(\mathbf{p}, \omega_{n}\right) \psi_{\downarrow}\left(-\mathbf{p},-\omega_{n}\right)\right\rangle,
$$

where

$$
g(\mathbf{p})=\cos \left(\frac{p_{x} a}{\hbar}\right)-\cos \left(\frac{p_{y} a}{\hbar}\right)
$$

is the $d$-wave form factor. Both theoretical and numerical lattice models indicate that the richness of the phase diagram results from a very delicate energy balance between the competing phases and is highly sensitive to the parameters of the theory and to external variables (e.g., temperature and doping) [13, [55].

Despite the differences in the nature and the scale of their interactions, QCD and high- $T_{c}$ materials resemble each other in significant ways. We already stressed that chiral symmetry is restored abruptly as the quark chemical potential is increased just as antiferromagnetic order vanishes in the presence of a sufficiently strong magnetic field. In each case, the varying external field is not coupled to the order parameter directly but rather 'exhausts' enough degrees of freedom to destroy the initial order. For each of these systems, we allow for the additional possibility of a thermodynamic competition between pairing and chiral/antiferromagnetic order. A major distinction between these two systems is that only two colors participate in the pairing state of QCD while all spins contribute to the $d$-wave order parameter of high- $T_{c}$ materials.

Random matrix models can be constructed as indicated in section 2, starting with the identification of the relevant symmetries and a construction of the interactions. 
For QCD, the additional color and spin symmetries need to be included in the matrix elements: The block matrix $W$ in equation (2.7) acquires an expanded color and spin block structure and becomes

$$
W=\sum_{\mu=0}^{3} \sum_{a=1}^{8} \lambda_{a} \otimes \sigma_{\mu} \otimes A_{\mu a}
$$

where $\sigma_{\mu}=(1, i \boldsymbol{\sigma})$ with $\boldsymbol{\sigma}$ the spin Pauli matrices, $\lambda_{a}$ are the generators of the color $S U(3)$ algebra, while $A_{\mu a}$ are real matrices representing single-gluon exchange. As before, ensembles averages are carried over the gluon field configurations; the matrix elements of $A_{\mu a}$ are drawn on a Gaussian distribution with zero mean. To respect Lorentz and color invariances in vacuum, the inverse variance is chosen to be independent of both indices $\mu$ and $a$ [32, 33].

The model for high- $T_{c}$ superconductivity is constructed in a manner similar to the phonon-exchange model of section 2.2. First, a parametrization is introduced that partitions the first Brillouin zone into states related by momentum reversal. In order to accommodate correlations induced by the AF state of equation (3.2), a further division is made between states that are related to one another by a momentum shift of $\mathbf{Q}=(\pi / a, \pi / a)$. Overall, this gives a total of four momentum regions, $\left(\mathbf{p}, \omega_{n}\right)$, $\left(\mathbf{p}+\mathbf{Q}, \omega_{n}\right),\left(-\mathbf{p},-\omega_{n}\right)$, and $\left(-\mathbf{p}-\mathbf{Q},-\omega_{n}\right)$, which partition the first Brillouin zone in four equal parts [37]. With this parametrization, the AF order parameter is written as

$$
\mathbf{m}_{A F}=\left\langle\sum_{r, s=1}^{4} \sum_{i, j=1}^{M} \sum_{\alpha, \beta=\uparrow, \downarrow} \psi_{r, i, \alpha}^{\dagger} \boldsymbol{\sigma}_{\alpha \beta}\left(\Gamma_{A F}\right)_{r, s} \delta_{i j} \psi_{s, j, \beta}\right\rangle,
$$

where $r$ and $s$ are region indices and $\Gamma_{A F}$ is the matrix

$$
\Gamma_{A F}=\left(\begin{array}{cccc}
0 & 1 & 0 & 0 \\
1 & 0 & 0 & 0 \\
0 & 0 & 0 & 1 \\
0 & 0 & 1 & 0
\end{array}\right) .
$$

In constructing the superconducting order parameter, we choose to replace the exact form factor $g$ by the low-energy approximation

$$
\phi_{d}(\mathbf{p})=\operatorname{sign}(g(\mathbf{p}))
$$

which neglects the detailed momentum dependence of $g$ but retains its $d$-wave character by changing sign for every 90 degree rotation of $\mathbf{p}$. The partition of the first Brillouin zone can be chosen so that $g$ is constant in each region [37]. The $\mathrm{SC}$ order parameter can then be written as

$$
m_{S C-d}=\left\langle\sum_{r, s=1}^{4} \sum_{i, j=1}^{M} \psi_{r, i, \uparrow}\left(\Gamma_{S C-d}\right)_{r, s} \delta_{i j} \psi_{s, j, \downarrow}\right\rangle
$$

where the $d$-wave form factor is given as

$$
\Gamma_{S C-d}=\left(\begin{array}{cccc}
0 & 0 & -1 & 0 \\
0 & 0 & 0 & 1 \\
-1 & 0 & 0 & 0 \\
0 & 1 & 0 & 0
\end{array}\right)
$$


The construction of the random matrix interactions is not as straightforward as in QCD, because there is no microscopic theory which formulates the problem in a form similar to that of the Yang-Mills action of equation (2.2). The problem could then be approached by writing an effective two-body potential at the level of the quartic term $Y$ of equation (2.11). The question is to identify the most effective two-body potential which accurately describes the interactions between the lowest-lying degrees of freedom in the system. This is for instance the approach followed by the Hubbard or the t-J model. We adopted a quite different approach by starting at the deeper microscopic level of the action, where we postulated the existence of a generic interaction carried by the exchange of either density or spin fluctuations. In some sense, this would describe a 'glue' interaction where Lorentz invariance is broken but rotational symmetry is preserved. Following the spirit of the models discussed above, no further specifications are made on the dynamics of interactions, whose matrix elements are drawn again on Gaussian distributions. A last, but not essential, element of the construction is the addition in the action of a spin-independent term $\psi^{\dagger} \Gamma_{t} \psi$ with

$$
\Gamma_{t}=\operatorname{diag}(t,-t) \otimes\left(\begin{array}{cccc}
1 & 0 & 0 & 0 \\
0 & -1 & 0 & 0 \\
0 & 0 & 1 & 0 \\
0 & 0 & 0 & -1
\end{array}\right),
$$

which mimics nearest neighbor hopping. Diagonal terms can be positive or negative, as are the free electron energies $\varepsilon(\mathbf{p})=-2 t_{0}\left(\cos \left(p_{x} a / \hbar\right)+\cos \left(p_{y} a / \hbar\right)\right)$. Similar to the nesting condition $\varepsilon(\mathbf{p}+\mathbf{Q})=-\varepsilon(\mathbf{p})$, the elements of $\Gamma_{t}$ change sign under a change of region corresponding to a momentum shift $\mathbf{Q}$ [37].

As constructed, the QCD and high- $T_{c}$ models can be solved exactly in the thermodynamic limit by following the methods described in section 2. Not surprisingly, the associated thermodynamic potentials have a very similar structure. Working again with the smallest Matsubara terms, we find the generic form

$$
\Omega(\sigma, \Delta)=A|\Delta|^{2}+B \sigma^{2}-\sum_{\ell, \pm} \log \left(E_{\ell \pm}^{2}+\pi^{2} T^{2}\right),
$$

This result is quite remarkable: Even though underlying microscopic physics is very different in each system, the resulting potentials share a common structure. In fact, the models simplify greatly as the calculations proceed. Starting from a complicated block structure and many different variances at the level of the action, the final result depends only on the two constants $A$ and $B$, which scale linearly with the variances [32, 33, 37]. In equation (3.12), $\Delta$ are pairing fields while $\sigma$ denotes either the chiral or the AF field. The $E_{\ell \pm}$ are the quasifermion energies in a fixed background of $\sigma$ and $\Delta$. In $\mathrm{QCD}$, the sum over $\ell$ is carried over the three colors. As only two colors participate in pairing, we have two ungapped excitations of energy $E_{ \pm}= \pm(\sigma+m)-\mu$ (as before in equation (2.17)) and four gapped excitations of energy $E_{ \pm}=\left[((\sigma+m) \mp \mu)^{2}+|\Delta|^{2}\right]^{1 / 2}$. For high- $T_{c}$, all excitations are gapped and have an energy $E_{ \pm}=\left[\left(\sqrt{t^{2}+\sigma^{2}} \mp \mu\right)^{2}+|\Delta|^{2}\right]^{1 / 2}$. These expressions are similar to the 
quasiparticle energies that were found in the microscopic theory of Ref. [56], and which can be written as $E_{ \pm}(p)=\left[\left(\sqrt{\varepsilon(p)^{2}+\sigma^{2}} \mp \mu\right)^{2}+g(p)^{2}|\Delta|^{2}\right]^{1 / 2}$, where $g(p)$ is the form factor given in (3.4). Approximating $\varepsilon(p)$ by $\pm t$ and $g(p)$ by $\phi_{d}(p)$ as in (3.8), so that $g^{2} \approx \phi^{2}=1$, the quasiparticle energies of the microscopic theory reduce to those of the random matrix theory.

The phase structures are readily derived from the two gap equations $\partial \Omega / \partial \sigma=0$ and $\partial \Omega / \partial \Delta=0$, which are polynomial in $\sigma$ and $\Delta$ and can thus be solved exactly. It is also interesting to note that it is possible to rescale all fields and external parameters by a multiplicative constant, with the result that the phase topology depends only on the single ratio of $\alpha=B / A$. In QCD (high- $T_{c}$ ), small values of $\alpha$ favor chiral symmetry breaking (AF order) and large ratios favor superconductivity.

For QCD, an interaction mediated by single-gluon exchange has a ratio $B / A=3 / 4$. The corresponding phase diagram is shown in figure 1-(b). In addition to the chiral phase structure obtained in the model of section 2, a superconducting phase (SC) is found at the low-temperature/high density end of the plot. The transition is first order as the chiral broken (CB) and SC phases cannot be directly related to one another by a continuous transformation. It also is interesting to see how the phase structure evolves when $\alpha$ is varied away from the value $\alpha=3 / 4$ associated with QCD. In total, only six different topologies can be realized as shown in figure 1 and the phase structure of figure 1 -(b) is found over a large range of values of $\alpha$, i.e. $0.42<\alpha<1.05$. It can thus be concluded that this phase structure, which we associated with QCD, is fairly robust against moderate variations in the detailed description of the random matrix interactions. The random matrix results are consistent with those of early microscopic models of color superconductivity [6, 57]. Some microscopic models also found a wedge of mixed broken symmetry extending over the small temperature end of the first-order line, as shown in figure1 (c) [58, 59]. Note, however, that this phase structure is observed in the present model over a fairly small range of values of $\alpha$, i.e. $1.05<\alpha<1.06$. It thus appears to be more fragile than the structure of figure 1 (b). The robustness of the coexistence phase is also discussed in the context of NJL models in 60].

A similar investigation can be performed for the high- $T_{c}$ model. Here, in the absence of a detailed theory at the level of the action, the value of $\alpha$ is not known a priori. In order to restrict the size of the parameter space to be explored, we make a number of assumptions [37]: First, an AF state is expected at half-filling $\mu=0$. This leads us to force fluctuation exchanges to be stronger for spin than for density. Second, we consider interactions that are attractive in the $d$-wave channel but repulsive for $s$-wave pairing. This requires interactions that favor large momentum exchange of order $\sim \mathrm{Q}$. The two desired properties can be realised simultaneously with an appropriate choice for the variances of the random matrix interactions [37. For a given material, the variances must reflect the different strengths of the scattering elements between the four regions of states in the first Brillouin zone. Once the two conditions above have been imposed, the variances can still be varied over a continuous - but restricted — range of values. The resulting phase structure depends on the single parameter ratio $\alpha$, which can assume 


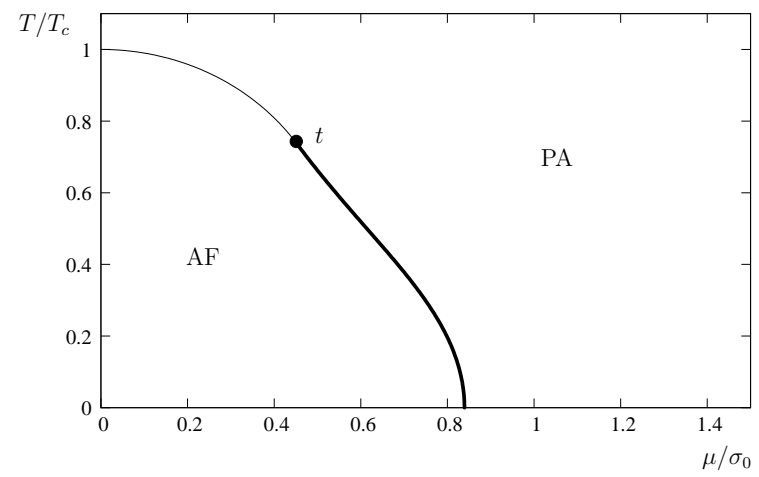

(a)

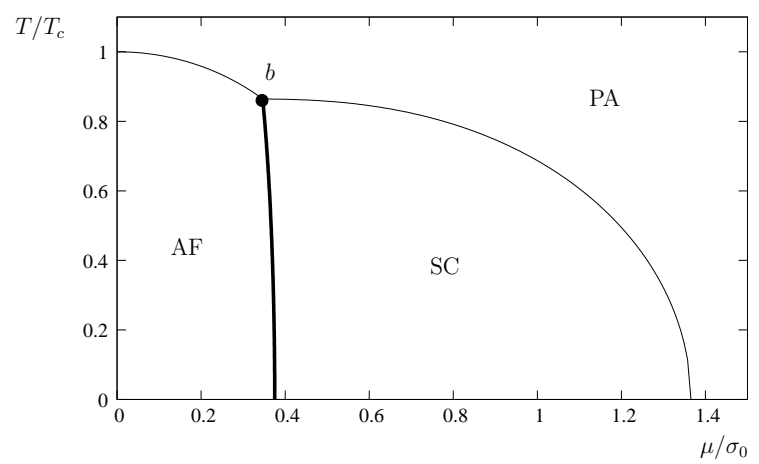

(c)

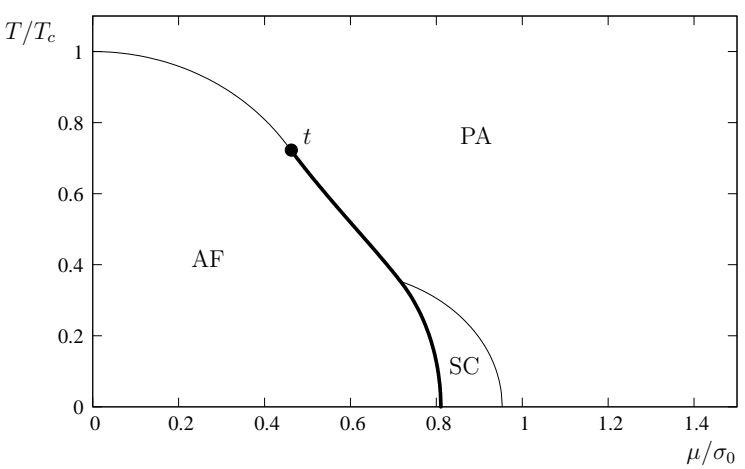

(b)

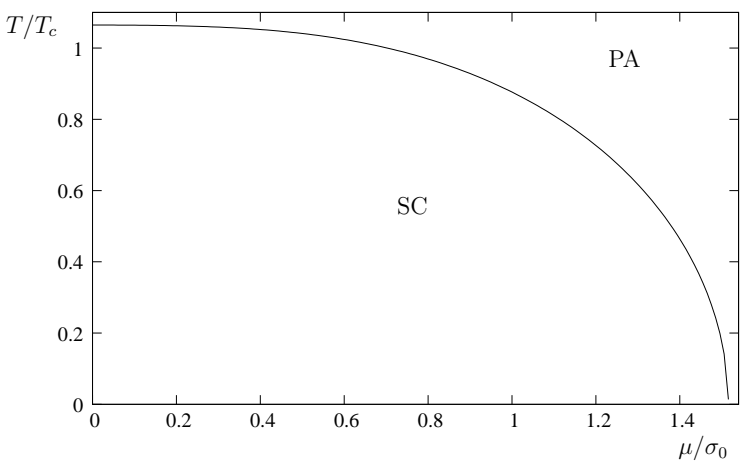

(d)

Figure 2. The four allowed topologies for the phase diagram of high- $T_{c}$ models. AF is the antiferromagnetic phase, PA the paramagnetic one, and SC the superconducting phase. First- and second-order transitions are depicted by thick and thin lines, respectively. $t$ is a tricritical point and $b$ is a bicritical one. $T_{c}$ is the transition temperature for $\mu=0$ and small $\alpha$ and $\sigma_{0}$ is the AF field at zero $\mu$. A hopping term $t=0.5 \sqrt{2 / B}$ is assumed. From top left to bottom right, the panels correspond to $\alpha=0.07, \alpha=0.2, \alpha=0.8$, and $\alpha=1.1$. The figures are adapted from [37.

any positive value. The many allowed possibilities for the variances reduce in this case to only four distinct phase topologies, which are illustrated in figure 2 .

How well do these phase structures compare to experiments? High- $T_{c}$ cuprates exhibit an insulating AF phase at half-filling $(\mu=0)$, with an AF ordering that weakens as a function of doping and eventually disappears in favor of an SC phase with a domelike phase boundary (See, e.g., [14, 61, 62]). Figures 2 (b) and (c) already exhibit the coarse structure of this picture. However, a closer analysis reveals several discrepancies. First, the present random matrix approach does not distinguish electronfrom hole-doped materials. This can be traced back to the kinetic energy term, which takes only nearest-neighbor hopping into account and exhibits the nesting property. As a result, the thermodynamic potential is an even function of $\mu$. In principle, nextnearest neighbor and higher-order hopping terms could be added to the model by 
using a hopping matrix whose eigenvalues have a sign distribution that reproduces the symmetries of the corresponding kinetic energies in the various regions of the Brillouin zone. Although we have limited ourselves to nearest-neighbor terms here, we expect that a model with a more elaborate hopping matrix would distinguish between electron and hole dopings. The second discrepancy concerns the transition between AF and SC phases, which is reported to occur either through phase separation, structured spin and charge distributions, or via a mixed $\mathrm{AF}+\mathrm{SC}$ phase. (E.g., see the discussion in 62 and references therein.) Here, only the first scenario of a first-order transition is found in all cases. A third discrepancy can be observed in the absence (for hole doped materials) of a pseudogap region separating the $\mathrm{AF}$ and $\mathrm{SC}$ phases. We will make further comments on the last two discrepancies in the end of this section.

Returning to the similarities between the random matrix models for QCD and high$T_{c}$ materials, limitations regarding the extension of some phases can be found in both cases. In QCD, a microscopic theory would contain the same logarithmic singularity as that present in the right side of equation (2.23) so that the diquark phase would extend to arbitrarily high $\mu$. In the high- $T_{c}$ model, a similar singularity exists in the gap equation for AF [63, 64] and results in an antiferromagnetic phase that exists independent of the hopping strength $t$. In the random matrix model, by contrast, $t$ must be kept below a threshold value for the AF to be realized [37]. As discussed earlier in subsection 2.2. these discrepancies are acceptable in a mean-field approach as they concern situations where the condensates are weak and thus fragile.

A striking difference can be observed in the thermodynamic competition between the order parameters when comparing the diagrams in figures 11 and 2 . Both types of systems support the coexistence of different phases along a first-order line. The two competing phases then coexist at a given temperature with different densities. They form an inhomogeneous state whose detailed spatial structure depends on the energetic cost of creating an interface. Clearly, such a description is beyond the simple approach considered here. A completely different situation occurs for the quark systems only. As $\alpha$ is increased in favor of color-superconductivity (with values that are no longer representative of $\mathrm{QCD}$ ), a tetracritical point — the meeting point of four different continuous transition lines - appears. The transition from one phase to the other then proceeds continuously through an intermediate phase of mixed broken symmetry, where both orders coexist in a spatially homogeneous phase. For the high- $T_{c}$ system, such a mixed broken symmetry state is absent. At best, a bicritical point appears, marking the end of a first-order line with its discontinuous transition.

The absence of a tetracritical point in the high- $T_{c}$ case is a direct result of the absence of ungapped excitations. The conditions for the observation of a tetracritical point can be determined by expanding the thermodynamic potential of equation (3.12) as a power series

$$
\Omega(\sigma, \Delta) \approx \Omega(0,0)+a_{2} \sigma^{2}+b_{2}|\Delta|^{2}+a_{4} \sigma^{4}+b_{4}|\Delta|^{4}+c_{4} \sigma^{2}|\Delta|^{2}+\ldots,(3
$$

where the coefficients are known functions of $\mu$ and $T$. The AF state makes a transition 
to a paramagnetic state along the line $a_{2}=0$, whereas the superconducting gap vanishes along the second-order line $b_{2}=0$. A critical point arises at the crossing of those two lines. A mixed broken symmetry phase (with non-zero values of both $\sigma$ and $\Delta$ ) can be stable near this critical point provided that the coefficients of the quartic terms satisfy the inequality $\rho_{4}=4 a_{4} b_{4}-c_{4}^{2}>0$ [37. In this case, the critical point is a tetracrictical one. In the opposite case, $\rho_{4}<0$, the critical point is only bicritical. For the high- $T_{c}$ model, it can be shown that $\rho_{4}<0$ for all values of $\alpha$. If a small fraction, $f$, of the excitations were forced to be ungapped, $\rho_{4}$ would become positive for a range of values of $\alpha$ near unity, and a tetracritical point would be present for those couplings.

Although the mixed broken symmetry phase is never realized as a global minimum of the thermodynamic potential of the high- $T_{c}$ model, it is always an unstable solution of the gap equations, i.e., a saddle-point of $\Omega(\sigma, \Delta)$. Further, the difference in energy between this state and the global minimum of $\Omega(\sigma, \Delta)$ is small on the scale of the inverse variance. This suggests that the existence of a mixed broken symmetry phase is a delicate matter. It may well occur in specific dynamic models or in calculations more detailed than those presented here, e.g., calculations beyond mean-field level, but the demonstration that its appearance is robust may be challenging. This is an example of useful information that can be obtained from a random matrix approach even though a precise assessment of those effects requires more than the mean-field treatment of random matrix theory.

A question related to the occurrence of critical points is whether the theory exhibits, approximately or exactly, condensation patterns of higher symmetry near these points. This would be the case, for instance, if the series expansion of $\Omega(\sigma, \Delta)$ could be expressed as a polynomial in a single variable, i.e., a linear combination of $\sigma^{2}$ and $\Delta^{2}$. In such a case, the different phase transitions could be described as a rotation of a metavector order parameter constructed with both $\sigma$ and $\Delta$, in the manner of the $\mathrm{SO}(5)$ theory of superconductivity of Zhang [65] or the $\mathrm{O}(10)$ theories that have been proposed for QCD [66]. We have found no indications of such higher symmetry in the vicinity of a critical point for either the quark or the high-Tc systems described here. There is one interesting case: In the high- $T_{c}$ model in the limit $\mu=0$, the potential of equation (3.12) becomes a function of the combination $\sigma^{2}+\Delta^{2}$ provided that $\alpha=B / A=1$. This higher symmetry is manifest only at $\mu=0$ and is broken for $\mu>0$ in favor of an SC ground state.

We end this discussion with some comments on possible extensions of the model for high- $T_{c}$ superconductors. The present construction is based on only a few elements, and we have shown how it can help in identifying symmetry-related results. At the same time, the model cannot be expected to describe the entire spectrum of materials, which can greatly differ in their detailed microscopic interactions. We have noted that some of this variety can be encoded in the choices for the variances to be associated with scattering processes between the different states of the first Brillouin zone. The strength of these matrix elements can most likely be probed by two-point correlation functions such as spin or density susceptibilities, which are experimentally accessible. Further 
work is needed in this direction. We also mentioned earlier the lack of a pseudogap phase in the random matrix phase diagram. A pseudogap region is thought to arise as a result either of correlations competing with the AF and SC orders or from preformed Cooper pairs leading to large phase fluctuations in the pairing field [14]. We have not attempted to model the pseudogap phenomenon here but can offer tentative indications of the effort that would be required in this direction: The scenario of a competing order could in principle be taken into account by decomposing the four-fermion random matrix potential $Y$ simultaneaously into the $\mathrm{AF}, \mathrm{SC}$, and the pseudogap channels (provided one can make meaningful "sign approximations" similar to those of the SC channel) and by constructing a new thermodynamic potential. The difficulties that could arise in this approach would be the lack of a true density of states distributed around a Fermi sea. This would make it difficult to obtain a faithful description of the Fermi arc phenomenology but might still capture some of the interesting physics. The scenario of phase fluctuations is less easily addressed in the present approach, since the degrees of freedom associated with the fluctuations need to be introduced explicitly in the model.

\section{Extension to a model for the ferropnictides}

As an example of a possible extension of the models discussed so far, we turn to the recently discovered ferropnictides, which constitute another class of type-II superconducting materials exhibiting magnetic and superconducting correlations. A current discussion of the iron arsenide compounds concerns the symmetry of the pairing state and its relationship to the phase diagram. It has been claimed by several authors [18, 19, 20, 21] that the occurrence of a mixed broken symmetry phase would constitute an evidence for a pairing with an $s_{+-}$symmetry, as opposed to the conventional $s_{++}$-wave. This claim relies on the observation that $s_{+-}$pairing leads to a mixed broken symmetry state if the two following conditions are met: The system has a finite chemical potential breaking the particle-hole symmetry, and the electron band is elliptic whereas the hole band is circular. In contrast, an $s_{++}$state meeting these two conditions does not lead to a mixed broken symmetry state [18, 19].

The main elements of the corresponding microscopic theory are the following [67]: The electronic structure of the system shows two hole pockets at the center of the first Brillouin zone and electron pockets shifted at either $\pm(\pi, 0)$ or $\pm(0, \pi)$ (for a square lattice with one Fe atom per cell). An AF state may develop with a form similar to equation (3.2), where the sum over states $\{\mathbf{p}\}$ is now carried over one type of pocket, whereas the corresponding $\{\mathbf{p}+\mathbf{Q}\}$ states with $\mathbf{Q}=(\pi, 0)$ belong to the other type of pocket. In the $s_{+-}$pairing state, the gap function changes sign from the hole pockets to the electron pockets, whereas an $s_{++}$gap function assumes the same sign in all pockets.

It is of interest to see how the model presented in the previous section should be modified to describe such materials. Following [19], we assume two electronic bands

shifted by the momentum $\mathbf{Q}$ with respect to each other. One can easily partition the first Brillouin in four equivalent regions related to one another by a shift in $\mathrm{Q}$ or by 
momentum reflection. The AF correlator then takes the same form given in equation (3.7). Similarly, the sign change that is characteristic of an $s_{ \pm}$-wave state turns out to be represented correctly by the matrix in (3.10). Finally, by collapsing each of the electron and hole bands into a single energy term, e.g., $t_{1}$ for electrons and $-t_{1}$ for holes,

the corresponding single-fermion Hamiltonian is now described by a term $\psi^{\dagger} \Gamma_{t} \psi$, where $\Gamma_{t}$ is given in (3.11) with the term $\operatorname{diag}(t,-t)$ replaced by a simple multiplicative factor, $t_{1}$.

By postulating random matrix interactions of the same form as in the model of the previous section, the resulting thermodynamic potential is precisely that of equation (3.12), for which all excitations are gapped. Thus, the phase diagrams are the same as those for the cuprates, and the random matrix model would preclude the occurrence of a mixed broken symmetry state. This is not inconsistent with the conclusions of [18, 19] since only one of two required conditions are met by the random matrix interactions. Here the model ignores the ellipticity of the electron bands. However, according to the discussion of the previous section, a mixed broken symmetry state might arise if some of the excitations do not participate in pairing, i.e. a fraction of the excitations are ungapped.

Two more results can be derived: First, following [19] and assuming equal coefficients for the quadratic terms of equation (3.12), i.e., $A=B$, one finds that the critical point $b$ is located on the $\mu=0$ axis and has a vanishing coefficent $\rho_{4}=4 a_{4} b_{4}-c_{4}^{2}$. This point is thus at the verge of becoming a tetracritical one. In contrast, assuming an $s_{++}$state and fixing $A=B$ yields a critical point which has $\rho_{4}<0$ and is thus bicritical. These results are consistent with the microsocopic model of [19].

The above findings demonstrate that the random matrix model already captures

many elements of the physics contained in the microscopic model of [19. It will be interesting to see whether the ellipticity of the electron states might also be mimicked in the random matrix approach and how it might affect the results.

\section{Conclusions}

\subsection{A summary}

We have reviewed the construction of random matrix models for the phase diagrams of a variety of systems. The general strategy starts at the level of the action with a description of the interactions between fermions and exchange fluctuation fields. These interactions assume the form of matrices with a global block structure dictated by the symmetries of the theory. The detailed form of the interactions is simplified materially by drawing matrix elements in the individual blocks at random. The result is a meanfield model which is exactly soluble. The corresponding thermodynamic potential has a simple structure involving only a minimal number of parameters and an explicit sum over Matsubara frequencies. This form is perfectly suitable for numerical purposes, but even greater simplification can be obtained by truncating this sum to a single term. 
While this truncation has quantitative consequences, it does not alter the topology of the resulting phases for the cases considered.

Despite - or even because of - the many simplifying approximations made, we believe that this approach to phase diagrams can be of value. In many calculations, it can be difficult to distinguish between solid results that are protected by the underlying symmetries of the problem and more tentative result that depend on the specific model investigated. By performing an ensemble average over theories, the results of the random matrix approach are dictated by symmetries alone. They can thus provide guidance in identifying model-dependent and model-independent features. Similarly, they can be useful in understanding the minimal number of symmetry constraints required to reproduce specific phase structures. The great simplicity of the thermodynamic potential obtained in these calculations makes it easy to check the robustness of predictions with respect to variations in the description of the interaction. We also note that near critical points, random matrix models bear strong similarities to GinsburgLandau theories with the advantage of the presence of additional constraints, which are inherited from the symmetries of the random matrix interactions. The freely-adjustable parameters of Ginsburg-Landau theory are replaced by known functions of temperature and chemical potential. As we have demonstrated above, these constraints can be extremely helpful in ruling out certain topologies in the phase diagram.

Difficulties associated with the thermodynamic limit can be formidable when calculating the phase diagram. The analysis in the previous sections showed that the partition function is exponentially small in the volume of the system. Moreover, the thermodynamic limit does not commute with other limits in the system. As a result, care must be taken in cases where low-energy metastable states exist, as their contribution to the partition function may be difficult to be distinguished from that of the ground state. In such circumstances, a random matrix model can be useful in identifying the metastable states. For systems with a finite fermion chemical potential, the situation can be significantly worse. For example, the QCD Dirac operator is complex as a consequence of the fermion sign problem, and numerical calculations with traditional sampling methods are precluded. In such cases, random matrix methods represent one of the few options available.

In the form presented here, random matrix models can be extended to any system where the fermions are the main degrees of freedom, the symmetries of the interaction are central to the problem, and the detailed dynamics of the exchange fields plays a secondary role. The model can be realized in either of two ways: The simplest approach consists of constructing the thermodynamic potential directly. This requires the identification of the auxiliary fields to be associated with the order parameters of the system and the determination of the single fermion energies in a fixed background of those fields. The remaining parameters could then be chosen freely. The richer approach adopted here is to start at the deeper level of the action. The reward for this extra effort is insight regarding the constraints that are imposed as a consequence of the symmetries of the interactions and their effect on the global properties of the system. 


\subsection{Open questions}

The various examples presented here indicate that a random matrices offer an interesting approach to the construction of phase diagrams. However, a number of open question remain:

- Fermion sign problem. Although the random matrix model can be solved at finite chemical potential — using a saddle-point method which becomes exact in the thermodynamic limit — numerical calculation of the same model are plagued by the fermion sign problem. Difficulties present at the microscopic level apparently disappear when working at a macroscopic level. The open question is thus to understand the resolution of this apparent contradiction and to see whether this mechanism can be exploited to ease the fermion sign problem. Considerable work in this direction has been performed in a QCD context (see, e.g., [68, 69, 70, 71, 72, 73]), where an interesting deterministic pattern in the sign oscillations of the fermion determinant has been seen.

- The nature of the interactions at the microscopic level. The random matrix model for high- $T_{c}$ was inspired by earlier models for QCD. Given this background, it was natural to identify the interactions at the more microscopic level as being mediated by the exchange of density and spin fluctuations. Is this structure generic, and can it represent other kinds of interactions, e.g., a static superexchange?

- Eigenvalue correlation for SC and AF states. In section 2.1 we noted that correlations due to chiral symmetry in QCD could be analyzed either at the microscopic level of the Dirac spectrum or at the level of the phase diagram. A similar analysis has not been performed either for the pairing state in QCD or for the $\mathrm{AF}$ and $\mathrm{SC}$ states in high- $T_{c}$ superconductivity. It would be of interest to see if the symmetries underlying these states induce similar microscopic correlations for a suitable single particle operator. Such an analysis might require the construction of an analogue of the Banks-Casher relationship.

- Summations over Matsubara frequencies. Since our primary concern has been to determine the physically realizable topologies of phase diagrams, we were willing to make simplifications in the thermodynamic potential which preserved their topology. This led us to truncate the infinite sum over Matsubara frequencies given by (2.17) by a single term. The resulting form, given by (2.18), is sufficiently simple that it can be studied 'by hand', but it is quantitatively different from the full result. It would be interesting to determine the extent to which the full random matrix result of (2.17) can provide a quantitative description of model systems for which the phase diagram can be constructed reliably. A related question is whether the inequality $\rho_{4} \leq 0$ for all $\alpha$, found for both high- $T_{c}$ and iron arsenide materials, holds for the full result. A direct comparison with the microscopic theory of [19] shows that $\rho_{4}$ has the same sign in both the random matrix approach and in the microscopic theory when $\alpha$ is near 1 . It would be interesting to see if this result remains valid in other cases. 
- Why do random matrix models work? We end this discussion by offering some admittedly speculative suggestions about the origin of the success of random matrix theory. Consider a real gas that obeys the ideal gas law. Interactions between its molecules are strong, but their role is strictly limited to the establishment of a statistical population of the states. The properties of the gas then follow from purely statistical arguments that are independent of the nature of the underlying molecular interactions. (Clearly, no one would consider attempting to determine the details of the molecular interaction from the ideal gas law.) Similar ideas form the basis of Niels Bohrs' compound nucleus model [74]. It is presumed that the energy of an incoming particle is rapidly distributed statistically among the constituent nucleons. The description of the eventual decay of the excited state is determined solely by this statistical distribution and is independent of the details of the formation process. This is directly related to Wigner's ideas that random matrix theory should apply whenever single-particle energy levels are strongly intermixed and contribute in an equilibrated or 'democratic' way to physical observables. To the extent that these notions can be extended to encompass the thermodynamic properties of QCD or high- $T_{c}$ materials, the decision to replace the exact action by an ensemble average should be legitimate. The phase diagram inherits only the symmetries of the underlying action and is independent of its detailed properties. Two additional clues point to the importance of strong energy level mixing. In 1984, Bohigas, Giannoni, and Schmit observed that the level correlations of the Sinai billiard - a quantum system with a chaotically behaved classical analog were consistent with the predictions of RMT (in this case, those of the Gaussian orthogonal ensemble). They further conjectured that RMT should correctly predict the spectral correlators of any system whose classical analog is chaotic [76]. Here, QCD and high- $T_{c}$ superconductivity are both described by non-linear field theories. The corresponding classical theories, and even more so the quantum many-body theories, are expected to be chaotic (i.e., ergodic) in nature.

A second indication for the need for energy mixing comes from observed limitations on the range of validity of the RMT description of spectral correlators. In principle, RMT is expected to provide an exact description of all spectral correlators on an energy scale which is vanishingly small compared to the full support of the spectrum. In practice, RMT predictions for many systems (including QCD) agree for eigenvalues with are smaller than the so-called Thouless energy, $E_{\text {Th }}$ (e.g., see [29]). This scale can be determined using ideas from localization theory, where $E_{\mathrm{Th}}$ is inversely proportional to the time required for a particle to diffuse across the system. For energies greater than $\sim E_{\text {Th }}$, a particle crosses the system in a time that is too short to resolve individual states. Given that the reflections at boundaries scatter the particle to other energy states, a large number of individual energy states end up contributing to the wave function, thus yielding the apparent 'democracy' underlying the RMT description.

Ultimately, a strong argument for the value of the random matrix approach to phase 
diagrams should rely on clues for universality on both microscopic and macroscopic scales. For a chiral random matrix model of QCD at zero $\mu$ and with a temperature dependence given by the two lowest Matsubara frequencies, it was shown that the thermodynamic approach and the Banks-Casher relationship yield exactly the same chiral condensate as a function of temperature [75]. These results strongly suggest that the physical content of the thermodynamic approach is actually dominated by the microscopic spectral density — which we believe to be universal. For $\mu>0$ and $T=0$, it was strongly argued that a random matrix model with the global symmetries of QCD yield a microscopic spectral density of the same form as that of the QCD Dirac operator [77, 78]. If similar connections can be established over most the $(\mu, T)$ plane and extended to a temperature dependence that includes all Matsubara frequencies, then one can expect that the phase diagrams that we constructed can be justified on the basis of the universality of the corresponding microscopic correlators. It would be of particular interest to see if there is a relation between the inequality for $\rho_{4}$ identified for the phase diagrams of superconducting materials and the properties of the microscopic correlators. This also indicates that there is considerable interest in studying the microscopic spectral properties of condensed matter systems!

One final open question remains: Why is the random matrix approach described here so rarely used in the study of phase diagrams? The answer probably lies in the fact that the starting form of the random matrix can be dauntingly complicated. For example, the block form of the random matrix appropriate for the description of the color and spin symmetries of QCD (3.5) is $6 \times 6$ and contains 32 component matrices. As we have attempted to explain, these complications disappear step-by-step as one follows the relatively direct path towards the thermodynamic potential of (2.17) and the even simpler (but topologically equivalent) form of (2.18). Given the model-independence of the final phase diagram and the merits of this approach relative to Ginsburg-Landau theory as noted above, we believe that the initial investment in constructing the random matrix is richly rewarded and can be recommended.

\section{References}

[1] HEMCGC and HTMCGC collaborations 1993 Nucl. Phys. B (Proc. Suppl.) 30315

[2] Halasz M A, Jackson A D, Shrock R E, Stephanov M A and Verbaarschot J J M 1998 Phys. Rev. D 58096007

[3] Barrois B 1977 Nucl. Phys. B129 390

[4] Frautschi S 1978 Proceedings of workshop on hadronic matter at extreme density (Erice) CALT68-701.

[5] Bailin D and Love A 1984 Phys. Rept. 107325

[6] Alford M, Rajagopal K and Wilczek F 1998 Phys. Lett. B422 247

[7] Rapp R, Schäfer T, Shuryak E V and Velkovsky M 1998 Phys. Rev. Lett. 8153

[8] Rajagopal K and Wilczek F (2000) at the Frontier of Particle Physics - Handbook of QCD (World Scientific, Singapore) chap. 35

[9] Itoh N 1970 Prog. Theor. Phys. 44291 
[10] Alford M G, Schmitt A, Rajagopal K and Schäfer T 2008 Rev. Mod. Phys. 801455

[11] Rajagopal K 1999 Nucl.Phys. A661 150-161

[12] Dagotto E 1994 Rev. Mod. Phys. 66763

[13] Scalapino D J 2007 Handbook of High-Temperature Superconductivity. Theory and Experiments (Springer, New York) chap. 13

[14] Lee P A, Nagaosa N and Wen X-G 2006 Rev. Mod. Phys. 7817

[15] Carlson E W, Emery V J, Kivelson S A and Orgad D Superconductivity 2008 (Springer, New York) chap. 21 p. 1225

[16] Ni N, Tillman M E, Yan J-Q, Kracher A, Hannahs S T, Bud'ko S L and Canfield P C 2008 Phys. Rev. B 78214515

[17] Chu J-H, Analytis J G, Kucharczyk C and Fisher I R 2009 Phys. Rev. B 79014506

[18] Fernandes R M, Pratt D K, Tian W, Zarestky J L, Kreyssig A, Nandi S, Kim M G, Thaler A, Ni N, Canfield P C, McQueeney R J, Schmalian J and Goldman A I 2010 Phys. Rev. B 81 140501(R)

[19] Fernandes R M and Schmalian J 2010 Phys. Rev. B 82014521

[20] Vavilov M G, Chukubov A V and Vorontsov A B 2010 Supercond. Sci. Technol. 23054011

[21] Vorontsov A B, Vavilov M G and Chukubov A V 2010 Phys. Rev. B 81174538

[22] Loh E Y, Gubernatis J E, Scalettar R T, White S R, Scalapino D J and Sugar R L 1990 Phys. Rev. B 419301

[23] Barbour I M, Morisson S E, Klepfish E G, Kogut J B and Lombardo M-P 1998 Nucl. Phys. Proc. Suppl. 60A 220

[24] Hands S 2007 Prog. Theor. Phys. Suppl. 168253

[25] Wigner E P 1951 Mathematical Proceedings of the Cambridge Philosophical Society 47 790-798

[26] Wigner E P 1955 Annals of Mathematics 62 548-564

[27] Wigner E P 1967 SIAM Review 9 1-23

[28] Forrester P J, Snaith N C and Verbaarschot J J M 2003 J. Phys. A:Math. Gen. 36 R1

[29] Guhr T, Mueller-Groeling A and Weidenmueller H A 1998 Phys. Rept. 299189

[30] Akemann G, Baik J and Di Francesco P (eds.) The Oxford Handbook of Random Matrix Theory (Oxford University Press, New York) in press

[31] Banks T and Casher A 1980 Nucl. Phys. B169 103

[32] Vanderheyden B and Jackson A D 2000 Phys. Rev. D 61076004

[33] Vanderheyden B and Jackson A D 2000 Phys. Rev. D 62094010

[34] Vanderheyden B and Jackson A D 2001 Phys. Rev. D 64074016

[35] Vanderheyden B and Jackson A D 2003 Phys. Rev. D 67085016

[36] Vanderheyden B and Jackson A D 2005 Phys. Rev. D 72016003

[37] Vanderheyden B and Jackson A D 2009 Phys. Rev. B 79144502

[38] t'Hooft G 1986 Phys. Rep. 142357

[39] Leutwyler H and Smilga S 1992 Phys. Rev. D 465607.

[40] Shuryak E V and Verbaarschot J J M 1993 Nucl. Phys. A560 306.

[41] Verbaarschot J J M 1994 Phys. Rev. Lett. 722531.

[42] J. J. M. Verbaarschot and I. Zahed, Phys. Rev. Lett. 70 (1993) 3852.

[43] Berbenni-Bitsch M E, Meyer S, Schäfer A, Verbaarschot J J M and Wettig T 1998 Phys. Rev. Lett. 801146.

[44] Berbenni-Bitsch M E, Meyer S and Wettig T 1998 Phys. Rev. D 58071502.

[45] Damgaard P H, Heller U M, Krasnitz A and Madsen T 1998 Phys. Lett. B 440129.

[46] Damgaard P H, Heller U M and Krasnitz A 1999 Phys. Lett. B 445366.

[47] Göckeler M, Hehl H, Rakow P E L, Schäfer A and Wettig T 1999 Phys. Rev. D 59094503.

[48] Damgaard P H, Edwards R G, Heller U M and Narayanan R 2000 Phys. Rev. D 61094503.

[49] Jackson A D and Verbaarschot J J M 1996 Phys. Rev. D 537223.

[50] Abrikosov A A, Gorkov L P, Dzyaloshinski E 1975 Methods of Quantum Field Theory in Statistical Physics (Dover Publications) 
[51] Bulut N and Scalapino D J 1991 Phys. Rev. Lett. 672898

[52] Monthoux P, Balatsky A and Pines D 1991 Phys. Rev. Lett. 673448

[53] Loram J W, Mirza K A, Cooper J R and Liang W Y 1993 Phys. Rev. Lett. 711740.

[54] Emery V J and Kivelson S A 1995 Nature 374434.

[55] Yao H, Tsai W.-F. and Kivelson S A 2007 Phys. Rev. B 76 161104(R)

[56] Kyung B 2000 Phys. Rev. B 629083

[57] Berges J and Rajagopal K 1999 Nucl. Phys. B538 215

[58] Kitazawa M, Koide T, Kunihiro T and Nemoto Y 2002 Prog. Theor. Phys. 108929

[59] Hatsuda T, Tachibana M, Yamamoto N and Baym G 2006 Phys. Rev. Lett. 97122001

[60] Buballa M 2005 Phys. Rep. 407205

[61] Damascelli A, Hussain Z and Shen Z-X 2003 Rev. Mod. Phys. 75473

[62] Demler E, Hanke W and Zhang S C 2004 Rev. Mod. Phys. 76909

[63] Hirsch J E 1985 Phys. Rev. B 314403

[64] Lin H Q and Hirsch J E 1987 Phys. Rev. B 353359

[65] Zhang S C 1997 Science 2751089

[66] Chandrasekharan S and Wiese U-J 2000 Preprint arXiv:hep-ph/0003214v1

[67] Mazin I I and Schmalian J 2009 Physica C 469614

[68] Stephanov M A 1996 Phys. Rev. Lett. 764472

[69] Splittorff K and Verbaarschot J J M 2007 Phys. Rev. D 75116003

[70] Han J and Stephanov M A 2008 Phys. Rev. D 78054507

[71] Osborn J C, Splittorff K, and Verbaarschot J J M 2008 Phys. Rev. D 78105006

[72] Bloch J and Wettig T 2009 JHEP 03100

[73] Andersen J O, Kyllingstad L T and Splittorff K 2010 JHEP 01055

[74] N. Bohr 1936 Nature 137344

[75] Jackson A D, Sener M K and Verbaarschot J J M 1997 Nucl. Phys. B 506612

[76] Bohigas O, Giannoni M J and Schmit C 1984 Phys. Rev. Lett. 521

[77] Osborn J C 2004 Phys. Rev. Lett. 93222001

[78] Akemann G, Osborn J C, Splittorff K and Verbaarschot J J M 2005 Nucl. Phys. B 712287 